\title{
Arte terapia para la escuela: una mirada hacia dentro y hacia fuera
}

\section{PREMIO CIMIED}

\author{
Art Therapy for Schools: Looking Inside and Outside
}

Versión en Español. *Full English Version at Anexo I

\author{
Alicia Santos Álvareza , Julia R. Gallego García ${ }^{b}$, M. Tránsito Domínguez Astorga ${ }^{c}$ y \\ Gonzalo Marcos Treceño ${ }^{d}$ \\ aCEO Camino de Santiago, La Virgen del Camino (León), asantosalva@educa.jcyl.es, , ${ }^{\mathrm{a}} \mathrm{DEAC}$ MUSAC, \\ León, julia@musac.es ${ }^{c} I E S$ Legio VII, León, mtdominguezas@educa.jcyl.e s y ${ }^{\mathrm{d} I E S}$ Legio VII, León, \\ gmarcos@educa.jcyl.es
}

\begin{abstract}
Resumen
La actual pandemia ocasionada por la crisis sanitaria derivada de la COVID-19 está suponiendo un reto para todos los profesionales que nos dedicamos a la educación. Cuatro Institutos de Educación Secundaria de la ciudad de León y su alfoz realizamos visitas escolares al Museo de Arte Contemporáneo de Castilla y León (MUSAC) online, a través de la plataforma Teams de Microsoft. Los estudiantes pueden visitar las exposiciones de forma telemática sin moverse del aula. En un trabajo conjunto con el Departamento de Educación y Acción Cultural (DEAC) del museo, enmarcado en un proyecto de investigación educativa, presentamos una manera de humanizar la enseñanza a distancia, trabajando con las emociones del alumnado para aumentar su motivación y actitud positiva hacia el aprendizaje. La investigación demuestra que el arte puede ayudar a superar los efectos emocionales de la pandemia.
\end{abstract}

Palabras clave: Innovación educativa, crisis COVID, visitas virtuales, TIC, colaboración institucional

\footnotetext{
Abstract

The current COVID-19 pandemic is challenging all professionals engaged in education. Four high schools from León make virtual visits to the Museum of Contemporary Art in Castilla y León (MUSAC) through Microsoft's Teams platform. Students can visit the exhibitions virtually, without leaving the classroom. Working together with the Department of Education and Culture (DEAC) on an educational research project, we present a way of humanizing distance learning, working with students' emotions to increase their motivation and positive attitudes toward learning. Studies show that art can help overcome the emotional effects of the pandemic.
} 
Arte terapia para la escuela: una mirada hacia dentro y hacia fuera

Keywords: Educational innovation, Coronavirus crisis, virtual visit, ICT, institutional collaboration 
Alicia Santos Álvarez, Julia R. Gallego García, M. Tránsito Domínguez Astorga y Gonzalo Marcos Treceño

\section{Introducción}

El presente trabajo se encuentra vinculado al Proyecto de Investigación Educativa titulado La práctica de las enseñanzas artísticas en tiempos de pandemia, financiado por la Consejería de Educación de Castilla y León a través de la Dirección General de Innovación y Formación del Profesorado ${ }^{1}$. Proyecto que surge de la necesidad de encontrar la manera de desarrollar todas esas actividades artísticas y culturales que, de forma habitual, se venían realizando en los centros educativos y se vieron frenadas a raíz de la actual crisis sanitaria.

Pero, ¿dónde encontramos el inicio? El germen de la experiencia que queremos compartir en este Congreso se encuentra en la webinar que desde el Departamento de Educación y Acción Cultural (DEAC) del Museo de Arte Contemporáneo de Castilla y León (MUSAC), con sede en León, programaron para presentar su oferta educativa a los docentes de la comunidad. Una webinar enmarcada al inicio de un curso escolar que arrancaba marcado por los rígidos protocolos que, desde la Consejería de Educación de Castilla y León, dictaron para evitar la propagación de la enfermedad causada por el virus SARS-CoV-2 y organizar así el regreso a la actividad lectiva presencial a los centros educativos de forma segura ${ }^{2}$. Entre estas medidas se encuentran el mantener una distancia mínima de seguridad de metro y medio entre las personas, la necesidad de trabajar con grupos reducidos de estudiantes, la no interacción entre alumnado de distintos grupos, el reducir en la medida de lo posible los desplazamientos fuera del centro, el no permitir la entrada al centro a cualquier persona ajena a los mismos o la suspensión de todas las actividades extraescolares y complementarias. Medidas todas ellas que dificultan la práctica de actividades que veníamos realizando en los centros educativos tales como la visita a los museos y que, a nuestro entender, empobrecen y deshumanizan tremendamente la práctica escolar.

Inmersos en este contexto de pandemia, desde el programa MUSAC ESCUELA del DEAC del MUSAC se pusieron a disposición de los docentes, tendiéndonos la mano para cualquier propuesta que quisiéramos transmitir, ofreciendo diferentes unidades didácticas en relación a sus exposiciones y/o recursos educativos, algunos de los cuales se habían creado a raíz de proyectos educativos o de investigación. El testigo fue recogido por tres de los docentes que asistimos a esa webinar, dando forma a este proyecto que involucra a cuatro centros educativos - tres Institutos de Educación Secundaria de la ciudad de León y su alfoz y un Centro de Educación Obligatoria-, trabajando en estrecha colaboración con el DEAC del MUSAC y contando con el asesoramiento científico del Departamento de Patrimonio Artístico y

\footnotetext{
${ }^{1}$ Esta comunicación analiza el trabajo conjunto que estamos desarrollando cuatro Institutos de Educación Secundaria de la ciudad de León y su alfoz, junto con el Departamento de Educación y Acción Cultural (DEAC) del MUSAC y el Departamento de Patrimonio Artístico y Documental de la Universidad de León. Esta experiencia se encuentra vinculada al Proyecto de Investigación Educativa otorgado por la Consejería de Educación de Castilla y León mediante ORDEN EDU/262/2020 de 9 de marzo (BOCYL de 17 de marzo) titulado La práctica de las enseñanzas artísticas en tiempos de pandemia.

2 Estas medidas se pueden entontrar en el Protocolo de prevención y organización del regreso a la actividad lectiva en los centros educativos de Castilla y León para el curso escolar 2020/2021, publicado por la Consejería de Educación de Castilla y León el 6 de julio de 2020, posteriormente adaptado al acuerdo 35/2020, de 16 de julio, de la Junta de Castilla y León y al acuerdo 49/2020, de 31 de agosto, de la Junta de Castilla y León.
} 
Documental de la Universidad de León. De esta manera, hacemos efectiva esa colaboración institucional real entre museo, escuela y universidad que, desde distintos ámbitos de la comunidad científica, se propone para una transformación constructiva de los sistemas educativos ${ }^{3}$.

Dada la temática del Congreso, de entre todas las actividades que componen el banco de recursos que estamos creando para poner a disposición del resto del profesorado, nos centraremos en exponer aquellas relacionadas con el DEAC del MUSAC, es decir, la forma en la que estamos llevando a cabo las visitas escolares al museo.

\section{Objetivos}

Para llevar a cabo nuestra propuesta, fijamos desde el inicio una serie de objetivos generales y específicos. Entre los primeros cabe mencionar los referidos a cómo ha influido emocional y académicamente el confinamiento y las restricciones derivadas de los protocolos educativos actuales sobre nuestro alumnado, a la vez que determinamos cómo el arte es capaz de influir positivamente en ellos, tanto a nivel personal como en su rendimiento escolar ${ }^{4}$. Queremos crear un banco de recursos educativos innovadores a disposición del profesorado y del alumnado, que sirva para la enseñanza de las materias propiamente artísticas, así como utilizar las artes como modelo pedagógico, mediante la introducción de dimensiones artísticas y culturales en otras disciplinas académicas.

Por lo que se refiere a los objetivos específicos, nos proponemos utilizar las TIC (plataformas online educativas, aplicaciones, redes sociales) como herramientas fundamentales que nos permitan salvar la distancia física impuesta en los distintos protocolos, aprovechando todo su potencial metodológico como recursos de gran motivación para el alumnado, que favorecen la enseñanza entre iguales o incluso la formación del docente a través de su alumnado ${ }^{5}$.

Será fundamental para ello construir la visita virtual al museo de una manera emotiva y participativa, que sirva de ventana al mundo exterior (introducir el placer de mirar, contemplar... como gesto muy humano y apaciguador que, además, nos permite trabajar el momento presente y la atención. Aprovechar para potenciar lo humano, generar calor, apoyo, momento de escucha y comprensión mutua para el autoconocimiento y entender que lo que nos está pasando es común a toda la comunidad educativa. Construir juntos resiliencias, aprender a dar la vuelta a las dificultades, convirtiéndolas en oportunidades de aprendizaje). Se trata de transformar el

\footnotetext{
3 A este respecto, Huerta (2008) afirma que "la investigación en aspectos de humanidades, arte y educación no se puede hacer en laboratorios ni a partir de medidas fijas o compartimentos estancos. La investigación que necesitamos ha de ser muy porosa a los flujos de ideas que traspira la sociedad. Los cambios son constantes y complejos" (pág. 16).

${ }^{4}$ Entre los primeros estudios sobre la afectacion emocional de la pandemia entre los adolescentes podemos citar a Mateu (2020) quien, en su artículo Cómo afecta emocionalmente la crisis del coronavirus a los adolescentes, señala la aparición de tristeza, ansiedad y problemas de sueño.

5 Muchos son los estudios que abordan este potencial didáctico y metodológico de las TIC. Podemos citar, a modo de ejemplo, a Priegue y Crespo (2011) quienes analizan "los beneficios que puede suponer la tecnología en el aprendizaje de los estudiantes haciendo especial hincapié no sólo en el potencial de estos soportes para favorecer la autonomía y responsabilidad del alumnado en el proceso de enseñanzaaprendizaje, sino también en sus posibilidades para el desarrollo de competencias de corte intercultural".
} 
Alicia Santos Álvarez, Julia R. Gallego García, M. Tránsito Domínguez Astorga y Gonzalo Marcos Treceño

concepto de museo, pasando del museo centrado en los objetos y en la información, a otro más abierto, donde las personas tengan voz e interactúen, donde se genere comunidad y prácticas transformadoras de las personas y las instituciones.

Partiendo de la experiencia vivida durante el confinamiento general de la población que tuvo lugar en marzo del pasado año, buscamos humanizar la enseñanza a distancia, trabajando con las emociones del alumnado para aumentar su motivación y actitud positiva hacia el aprendizaje.

De forma paralela, nuestro trabajo servirá para consolidar las enseñanzas artísticas como eje transversal del resto de las materias que integran el currículo de las enseñanzas oficiales de Castilla y León, incidiendo en el potencial multidisciplinar de las mismas y fomentando el pensamiento abstracto del alumnado para que, a través del arte, mejore cada una de las competencias clave que le ayuden a alcanzar un pleno desarrollo social, personal y profesional ${ }^{6}$. De esta manera, las artes se establecen como vía a través de la cual se hace efectiva una educación inclusiva, basándonos en un modelo educativo en el cual ningún alumno es dejado atrás, sino que todas sus cualidades y características incorporan riqueza, diversidad y una mirada poliédrica en relación a la educación artística, la creación y la persona (tanto docente como discente).

Por último, queremos fomentar la colaboración entre el profesorado de distintas materias y centros de la provincia de León?

\section{Desarrollo de la innovación}

Dado el carácter eminentemente práctico del proyecto de investigación que estamos desarrollando, todo el marco teórico sobre el que nos sustentamos está basado en la aplicación de lo que denominamos metodologías activas, entendiendo como tales "aquellos métodos, técnicas y estrategias que utiliza el docente para convertir el proceso de enseñanza en actividades que fomenten la participación activa del estudiante y lleven al aprendizaje”. (Labrador y Andreu, 2008). Entre estas metodologías activas, el trabajo cooperativo resulta fundamental, no solo a nivel del alumnado sino también y, fundamentalmente, entre el profesorado ${ }^{8}$.

Dada la imposibilidad de salir con nuestro alumnado del centro educativo para disfrutar de las visitas museísticas, es el museo el que entra en el aula a través de vídeollamadas con la plataforma Teams de Microsoft. Se trata de una herramienta de gestión del aula que la Consejería

\footnotetext{
${ }^{6}$ En este sentido se expresan los Resultados de la II Conferencia Mundial de la UNESCO 2010 sobre la Educación Artísitca (Seúl 2010).

${ }^{7}$ Pozuelos (2021) señala a este respecto que, entre las iniciativas activadas en las escuelas a partir de la pandemia para dar respuestas a nuevas necesidades pedagógicas, se encuentra la innovación dentro del equipo docente "El tradicional hastío que provocan las repetitivas y formales reuniones docentes han dado paso a una franca colaboración entre iguales. Atender a las inmediatas demandas educativas y compartir conocimiento profesional son una realidad que ha cobrado presencia en abundantes grupos de docentes dispuestos a prestarse ayuda con objeto de dar respuesta a los innumerables requerimientos que esta insólita situación ha provocado".

8 Tal y como se señala en el documento elaborado por el Laboratorio de investigación educactiva Lab "La investigación sobre un nuevo modelo educativo, consensuado por un equipo docente, exige que los profesores colaboren en las tareas de diseño y desarrollo del currículo. Cuando se comparte una experiencia, se justifica y se debate sobre ella, se convierte en teoría compartida. De este modo, la cooperación puede constituirse en plataforma para la búsqueda de nuevas prácticas" (LAB: sin fecha).
} 
de Educación de Castilla y León incorporó al paquete Office 365 Educación, generalizándose su uso por parte de la comunidad educativa a raíz del confinamiento general de la población el pasado mes de marzo del año 2020. Tanto el profesorado como el alumnado tienen acceso gratuito a esta herramienta con su usuario del portal, mientras que aquellas personas ajenas a los centros educativos pueden entrar como invitadas.

De entre todas las opciones que nos ofrece Teams, para realizar estas visitas virtuales con el MUSAC, creamos un equipo por aula, en el que incluimos al alumnado, al docente y a la educadora del museo. Todo el alumnado puede participar de la actividad en tiempo real, incluso los que se encuentran confinados en sus domicilios por razones de la COVID-19 o por cualquier otra eventualidad. Esta herramienta nos permite tanto la realización de videollamadas, como compartir los trabajos y actividades realizadas, incluso en el momento en el que estamos conectados con el museo, con la posibilidad de un feedback continuo entre el museo y los estudiantes. Se crea así un vínculo afectivo entre la institución museística y la comunidad estudiantil, incorporando el museo como un espacio y un referente educativo de primer orden entre nuestro alumnado.

Como complemento a estas videollamadas, utilizamos como herramienta suplementaria un Cuaderno de viaje. Se trata de un diario personal que recoge las experiencias relacionadas con el MUSAC, incidiendo en el registro emocional y premiando cada visita individual con un reflejo positivo en la calificación de la materia. La portada de dichos cuadernos es la ganadora del concurso convocado entre los alumnos participantes de los cuatro centros educativos.

En cuanto a la metodología elegida para la recogida de datos consiste en una combinación de técnicas cuantitativas y cualitativas. Respecto a las primeras, recogemos los datos de las visitas virtuales realizadas y del alumnado participante en tablas que nos permiten un análisis comparativo de los datos respecto a cursos pasados. Cualitativamente, llevamos a cabo una evaluación tanto de la afectación emocional de la pandemia sobre el alumnado como de cada una de las sesiones con el museo.

Para realizar esta evaluación utilizamos la observación directa en el aula, con anotaciones del profesorado y del alumnado ${ }^{9}$, preguntas orales en clase y la realización de cuestionarios, los cuales están realizados a través de la herramienta Google Forms y posteriormente editados en el Grupo Teams de cada aula, de fácil distribución para los profesores y acceso para los alumnos. Estos cuestionarios se perfilan así como una útil metodología de recopilación de información que nos da una visión más amplia y compleja sobre las actividades realizadas que la obtenida solo a través de la observación. A través de la evaluación comprobamos el grado de aceptación de las actividades propuestas y la comprensión de lo explicado en el aula (centrándonos siempre en la consecución de un aprendizaje significativo), adaptando, en la medida de lo posible, las próximas visitas a sus gustos y sugerencias.

\footnotetext{
${ }^{9}$ A este respecto, hemos nombrado a dos alumnos observadores por aula, a modo de los sismógrafos u observores meteorológicos de la Bauhaus, cuya labor consiste en registrar sus impresiones sobre lo ocurrido durante las sesiones virtuales con el MUSAC. Una referencia a estos sismógrafos la encontramos en la tesis doctoral de J. Hervás, El camino hacia la arquitectura: las mujeres de la Bauhaus.
} 
Alicia Santos Álvarez, Julia R. Gallego García, M. Tránsito Domínguez Astorga y Gonzalo Marcos Treceño

\subsection{Trabajo desde el aula}

Los docentes implicados en el proyecto preparamos cuidadosamente, junto con la educadora del DEAC, los detalles de cada sesión, realizando varias reuniones telemáticas previas a la visita con nuestro alumnado. Con ello conseguimos entender la exposición, los objetivos que queremos conseguir y las propuestas de actividades a realizar desde las asignaturas de origen, es decir, Geografía, Historia, Música y Matemáticas. Si conocemos el mundo creativo de cada uno de de los artistas cuya obra vamos a descubrir, nos resulta mucho más fácil transmitírselo posteriormente a nuestro alumnado.

A la hora de programar estas visitas museísticas, nos topamos con la dificultad de encontrar un aula con recursos digitales disponibles, especialmente durante este curso escolar, en el que por motivos de reducción de ratios por grupo, los espacios disponibles en los centros se han reducido mucho. Es cierto que hay disponibilidad de utilizar pantallas y cañones portátiles, pero con el inconveniente de que no es operativo por el tiempo necesario para su puesta en funcionamiento. También es necesario poner solución y adaptar el tiempo necesario de una visita con el museo (aproximadamente hora y media) a las sesiones lectivas de los institutos, que vienen a ser de cincuenta minutos.

Una vez superadas las dificultades técnicas y logísticas, logramos que el MUSAC entre en el aula. Los alumnos de los cursos más bajos reciben a la educadora con aplausos, mientras que todos sin expeción aguardan con emoción la llegada de una invitada que se nos presenta sin mascarilla en el rostro.

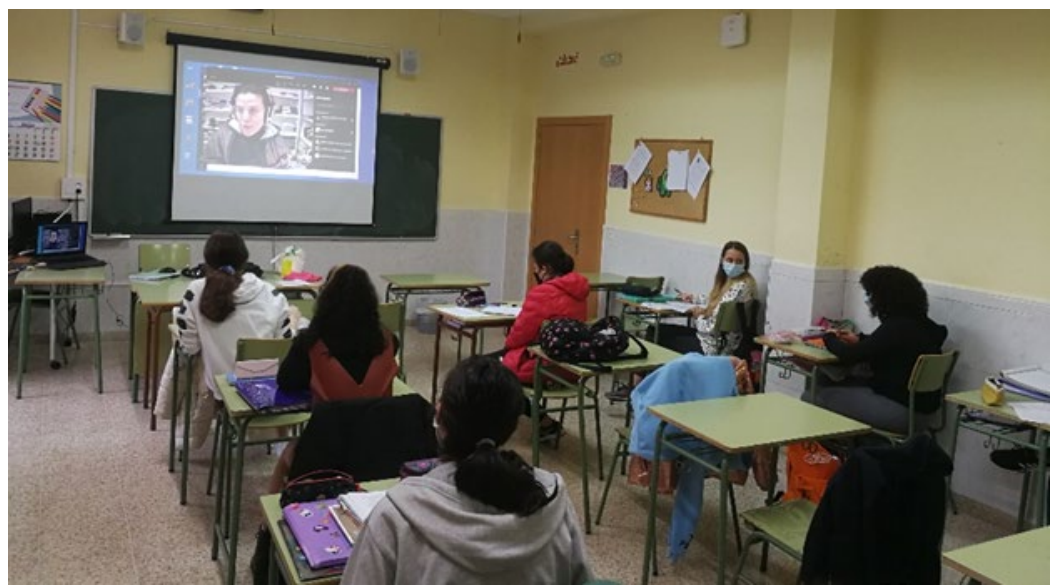

Fig. 1 EI MUSAC entra en el aula

A modo de ejemplo, desde la materia de Música hemos trabajado la diferencia entre Galería de Arte y Exposición de un Museo. Se ha contextualizado la Galería Tráfico de Arte de la ciudad en la que viven los alumnos, León. Se ha trabajado y visto la exposición del MUSAC, Tráfico de Arte. Galería, ciudad y periferia y, en concreto, la obra Felipe vuelve a casa con las ovejas sonando de Nilo Gallego. A partir de la exposición, de preguntas abiertas y de ir visitando las diferentes obras de esta exposición en la sala, se deriva hacia la tarea a realizar por el alumnado 
sobre el Paisaje Sonoro. Un paisaje sonoro personal de una actividad que realizan todos los días, ir de casa al instituto, poniendo en evidencia su presencia y su identidad como un ser único que crea unos sonidos y forma parte de un todo que no sería igual si no hubiera pasado por allí o dejara de hacerlo. Toman conciencia de lo que son como personas desde lo sonoro. Además son capaces de identificarse en el hecho de ser creadores y expresar a través de su grabación lo que llevan dentro al modo de un autor de arte contemporáneo como el trabajado en la sesión del MUSAC.

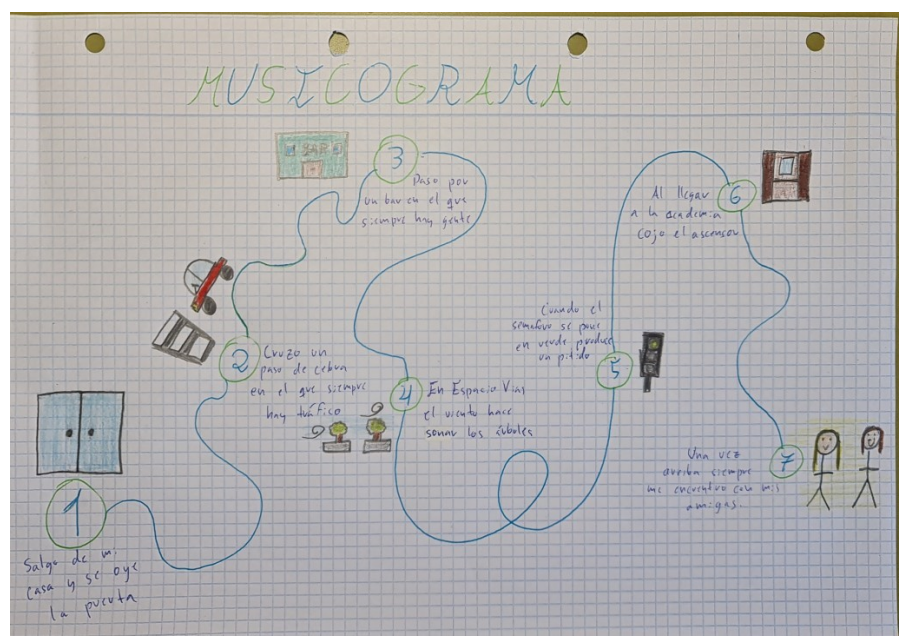

Fig. 2 Musicograma de paisaje sonoro elaborado por un alumno

\subsection{Trabajo desde el museo}

En los últimos veinte años hemos vivido un auténtico desarrollo en el campo de la educación en los museos. En el caso del DEAC, desde el principio nos volcamos en la escuela e intentamos desarrollar un programa escolar que no fuera solo, o no solo, visitas escolares al museo sin afectación. Queríamos crear vínculos con las instituciones, con los profesionales de la educación y con su alumnado (un público al que valoramos especialmente). Queríamos servir, apoyar, ayudar, traspasarnos mutuamente y transformarnos.

Siempre ha habido tanto trabajo o más en la trastienda de cada visita que casi en el durante, donde luego todo era disfrutar, porque era la esencia misma de nuestra labor... escuchar a la comunidad educativa, aprender de ellos... hacer juntos e intentar contribuir a la mejora del sistema educativo, por pequeña que fuera la aportación.

En el DEAC hemos realizado cursos de formación para el profesorado, seminarios, jornadas, equipos de trabajo, practicas educativas colaborativas, etc. Siempre buscando alianzas, compañeros al otro lado que quisieran embarcarse en la aventura del hecho educativo desde otros lugares. Siendo el museo otro recurso educativo más; no solo por sus exposiciones, sus instalaciones o sus medios. También por sus acciones, sus profesionales, sus saberes fruto de múltiples prácticas con gentes diversas que a diario nos influyen y nos dejan su huella. Y esto 
Alicia Santos Álvarez, Julia R. Gallego García, M. Tránsito Domínguez Astorga y Gonzalo Marcos Treceño

contribuye, más que nada, a un saber construido colectivamente y muy pegado a la realidad, a la calle... a los otros.

Esta parte de hermandad o interconexión ha sido nuestro principal objetivo todo este tiempo y fruto de ello han surgido proyectos como este, donde entendemos que lo que hacemos ayuda a que los alumnos tengan experiencias educativas de calidad, que no olviden y que, también, nos ayuden a transformarnos a nosotros mismos, igual que a ellos, para sentir que lo que hacemos contribuye a algo aun mayor; una educación más humana que propicie el desarrollo integral de todas las personas. Que nos permita, además, conoceros mejor y experimentar realmente la grandeza de eso llamado "hecho educativo".

Ese fue el motor de arranque de esta investigación, nuestra cuasi obsesión por llevar algo de "calor" a las clases, en estos tiempos de asepsia, de espacios desnudos de cualquier cosa susceptible de impedir una escrupulosa desinfección... sin carteles, ni dibujos, ni fotos,... sin apenas nada más, en las aulas tristes, pelonas y frías, con impersonales mesas y sillas verdes, siempre demasiado duras.

Sentíamos que entrar virtualmente desde el museo al aula descubriendo juntos sus exposiciones, sus artistas, sus obras, etc.... aportaban luz y color al aula. Pero no solo, además surgían emociones muy variadas con la contemplación y posterior conversación y reflexión grupal que suscitaba cada visita, percibiendo que los sentimientos y los afectos eran el principal motor que nos mantenía conectadas a ambas instituciones.

Y ver cuánto nos necesitábamos, no solo por hablar de arte y descubrir tantas cosas, tantos mundos, tantas ventanas por abrir, caminos nuevos... experiencias. Sino por tener un espacio para el desahogo, el descanso del miedo, la reflexión abierta y la libre expresión. Un espacio para pensar en voz alta y crear libremente, motivados por todo lo que las propuestas de los diferentes artistas removían en nosotros.

¿Cómo es el proceso de trabajo entre el museo y la escuela? Cada sesión, a partir de nuestras unidades didácticas, es diferente. Antes siempre hay un encuentro con cada docente para revisarla juntas, ajustarla y/o acomodarla a cada grupo, asignaturas o contenido de interés, en relación a lo que estén viendo en ese momento en el temario.

Después suele haber dos o tres videoconferencias (o sesiones de trabajo ya en el aula) por cada unidad didáctica. En la primera sesión nos conocemos, explicamos el proyecto (que ya ha sido también explicado previamente por cada docente) y visitamos la exposición que vamos a trabajar. Terminamos con un coloquio, o reflexión abierta de lo visto y planteamos una actividad creativa que les permita experimentar, desde la actividad creadora, y en cierta relación con el artista o artistas que hayamos descubierto en esa sesión.

En ocasiones hay un tiempo intermedio entre la primera y segunda sesión donde, tras la evaluación conjunta de cada sesión, intentamos solventar y reconducir aquellos problemas técnicos con los que nos hemos encontrado. Cuando es posible, algunos de estos handicaps los convertimos en oportunidades para el intercambio de roles, dado que el alumnado suele ser tan competente o más que nosotras... por otro lado, no dejan de ser estrategias propias de nuestra 
profesión habituada a trabajar con mucha precariedad. También, vemos si es necesario implementar con otros materiales y/o recursos adicionales que complementen la visita o favorezcan la práctica artística propuesta.

Lo acontecido en cada sesión de trabajo en el aula es registrado por el alumnado en su cuaderno de campo o porfolio personal cuya portada, por cierto, ha sido diseñada por ellos en el marco de un concurso de todo el alumnado, de los cuatro institutos integrantes en el proyecto. Este será un recurso para su autoevaluación. Además de una especie de pasaporte o diario donde reseñen sus visitas individuales al museo (tras la visita grupal por videoconferencia) y aquellas otras experiencias en relación que ellos/as consideren, siendo, todo ello objeto de valoración positiva en la evaluación global de la asignatura implicada.

En la siguiente sesión, los estudiantes nos muestras sus trabajos, investigaciones, propuestas artísticas, etc. Y entre todos compartimos las experiencias, aprendizajes y vivencias surgidas en el proceso y donde habitualmente se ven involucramos otros agentes del universo propio de cada alumno/a (por ejemplo, el proyecto Libertad realizado con $4^{\circ}$ de la ESO).

\section{Resultados}

Antes de analizar los resultados obtenidos hasta el momento, cabe recordar que el proyecto de investigación educativa que nos ocupa va a ser desarrollado durante los cursos académicos 2020/2021 y 2021/2022, por lo que los resultados aquí expuestos y analizados son solo una primera muestra, a la espera de los resultados finales.

En las visitas escolares al MUSAC han participado 148 escolares, pertenecientes a 7 grupos de Educación Secundaria Obligatoria de dos Institutos de Educación Secundaria y un Centro de Educación Obligatoria, con edades comprendidas entre los 12 y los 17 años.

En cuanto al número de visitas por grupo, la evolución respecto a curos anteriores ha sido la siguiente:

Tabla 1. Número de visitas escolares por centro y curso

\begin{tabular}{cccc}
\hline Curso escolar & $\begin{array}{c}\text { CEO CAMINO } \\
\text { DE SANTIAGO }\end{array}$ & IES LEGIO VII & $\begin{array}{c}\text { IES ANTONIO } \\
\text { GARCÍA BELLIDO }\end{array}$ \\
\hline $2018 / 2019$ & 1 & 1 & 2 \\
$2019 / 2020$ & 1 & 1 & 1 \\
(hasta el 13/03) & & & 0 \\
$2019 / 2020$ & 0 & 0 & \\
(confinamiento) & & & 2 \\
$2020 / 2021$ & 7 & 4 & \\
primer trimestre & & & \\
\hline
\end{tabular}


Alicia Santos Álvarez, Julia R. Gallego García, M. Tránsito Domínguez Astorga y Gonzalo Marcos Treceño

Al aplicar la herramienta de las videoconferencias, hemos aumentado considerablemente el número de visitas por curso, al multiplicarse estas por seis. Si durante un año académico estándar visitábamos el museo una vez por grupo y curso, con esta nueva forma de trabajar, hacemos una media de dos visitas trimestrales con cada uno de los grupos.

Respecto al alumnado participante en la actividad, hemos conseguido eliminar el alumnado absentista. Si bien durante otros cursos, con las salidas al museo programadas desde los centros escolares, un treinta y seis por ciento del alumnado no participaba en la actividad, con esta nueva forma de trabajar la participación alcanza el cien por cien. Incluso aquel alumnado que se encuentra confinado en sus domicilios participa en la visita en las mismas condiciones que su grupo de referencia.

Todas las visitas virtuales programadas se han realizado satisfactoriamente. Técnicamente, ha habido problemas de conexión inalámbrica en un cuarenta y tres por ciento de las mismas, tanto desde los centros educativos como desde el museo. Al realizar visitas en las que participan varias aulas de forma simultánea, en un doce por ciento de las mismas nos hemos encontrado con problemas de sonido: aunque se escucha desde las dos aulas, el museo solo recibe el audio de una de ellas, perdiendo el feedback con el alumnado de esa clase.

Aun así, el clima de trabajo ha sido excelente. Las visitas se han realizado en un ambiente óptimo para el estudio, con un alto grado de interés y motivación por parte del alumnado y del profesorado. No ha habido ningún caso de alumnos disruptivos que hayan dificultado el normal desarrollo de la actividad, consiguiendo una educación inclusiva, en la que nadie queda atrás.

Cada una de las visitas realizadas con el museo ha dado lugar a producciones individuales del alumnado, con propuestas didácticas que se han trabajado desde las áreas de Historia, Geografía, Música y Matemáticas.

Si analizamos el grado de satisfacción del alumnado, manifiestan su agradecimiento por realizar estas actividades y poder ir gratuitamente al museo a posteriori. Se sienten protagonistas de sus propias ideas "expresivo - artísticas" mostrándolas en el aula a sus compañeros y profesor de base, en las sesiones posteriores a la sesión con la educadora del museo.

La valoración que el alumnado participante en el proyecto hace respecto al arte contemporáneo también difiere respecto a los grupos que no participan en él: se identifican con el proceso de expresar y de crear, sin juicio. Conocen mejor los motivos y el impulso creador del artista contemporáneo. Respetan y valoran las exposiciones, sus obras y los autores.

De acuerdo con la metodología aplicada en el presente proyecto recogemos información de las actividades que se van realizando. Al alumnado le pedimos que haga una autoevaluación al final de cada actividad en forma de un cuestionario que funciona como un termómetro emocional para registrar el grado de bienestar y la valoración que hacen de las actividades en las que participan. A partir de un número de sentimientos recogidos en el cuestionario inicial, cada alumno los ordena según el grado predominante experimentado en una escala de mayor a menor. 
Tabla 2. Termómetro emocional para registrar el grado de bienestar y la valoración que hacen de las actividades en las que participan. (Enero 2021)

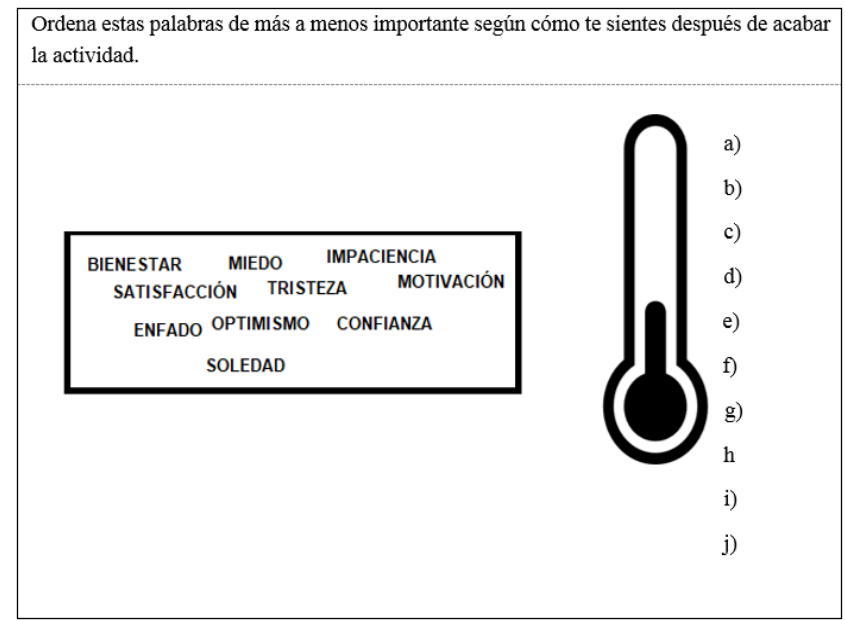

Así, los cuestionarios sobre la afectación emocional de la pandemia sobre el alumnado señalan que las emociones más recurrentes vividas durante el confinamiento fueron la tristeza, la soledad, el miedo, el enfado y la impaciencia. Este estado emocional se ha visto modificado con la vuelta a la actividad lectiva presencial y, tras el trabajo llevado a cabo junto con el MUSAC, los cuestionarios nos muestran que se van diluyendo en gran medida los sentimientos de miedo y soledad, aflorando otros como la confianza, la motivación, la satisfacción y el optimismo.

NOTA a TABLAS 2 y 3 - En este estudio, aceptamos todas las emociones, tratando de no juzgarlas y validando que cualquier emoción es permitida y posible. Para resumir y según condicionamiento social, las denominamos más negativas y positivas.

Tabla 3. Resultados Cuestionario alumnado relacionando su estado emocional con sus estudios al inicio de la pandemia (Septiembre 2020)

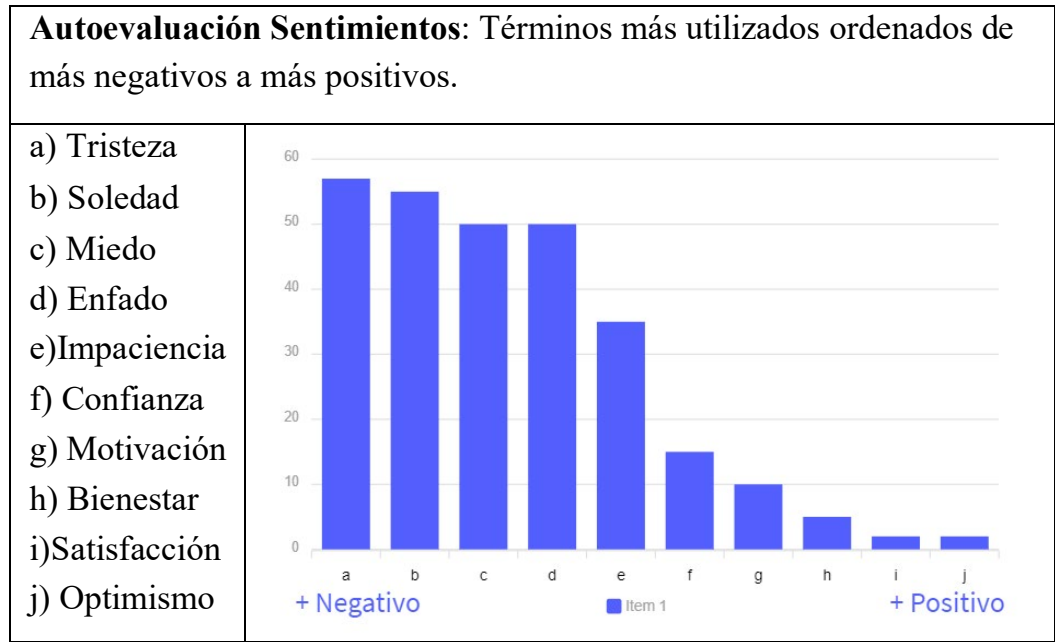


Alicia Santos Álvarez, Julia R. Gallego García, M. Tránsito Domínguez Astorga y Gonzalo Marcos Treceño

Tabla 4. Resultados Cuestionario Final $1^{\circ}$ Trimestre Alumnado relacionando su estado emocional con sus estudios incluyendo las actividades del Proyecto con el MUSAC (enero 2021)

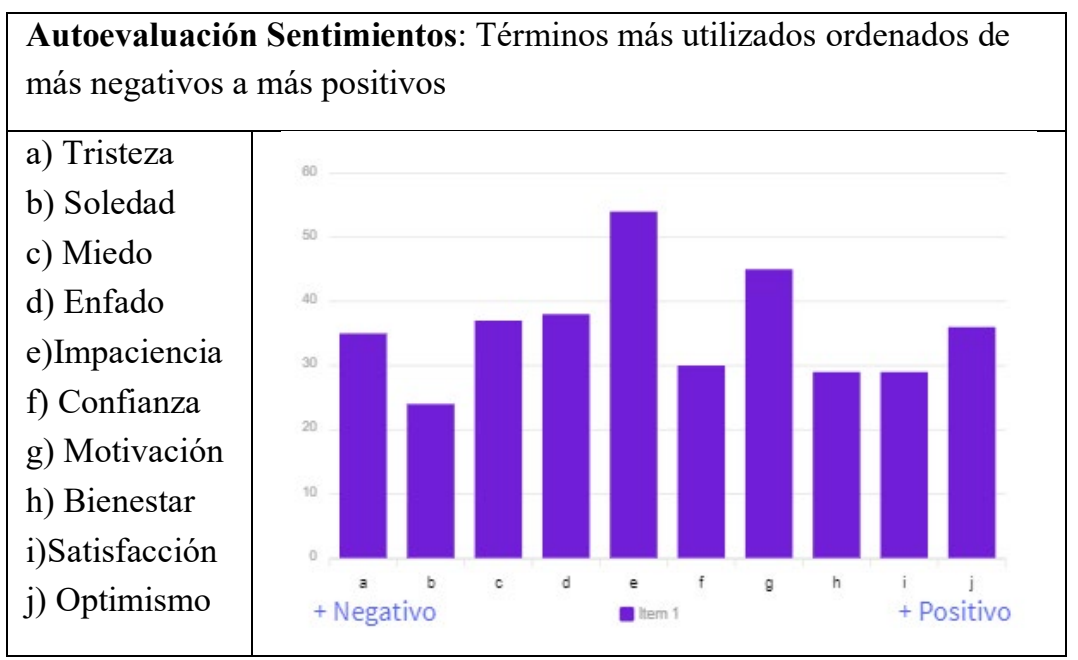

\section{Conclusiones}

A la vista de los resultados obtenidos, hemos comprobado que, a cualquier edad, el confinamiento agrava los problemas psicológicos, ya que el ser humano necesita de experiencias sociales. El arte sirve para expresar sentimientos difícilmente verbalizables y ayuda al alumnado a tomar conciencia de su estado y evolución emocional. Muchos de los alumnos manifiestan a través del cuestionario que han necesitado de actividades como tocar un instrumento, bailar, dibujar o escuchar música para sentirse mejor en los meses confinados. En las épocas difíciles, los alumnos han descubierto fortalezas escondidas en su personalidad, como la solidaridad, la empatía, las ganas de ser positivo, el apoyo afectivo a sus familias y amigos.

En los grupos en los que hemos trabajado junto con el MUSAC se ha reducido el nivel de estrés entre el alumnado, con un menor grado de ansiedad frente a los exámenes y una mayor confianza en sus capacidades individuales. Del mismo modo, observamos que la gran mayoría visita posteriormente de forma presencial el museo. A este respecto, el Cuaderno de viaje ha servido para potenciar las visitas individuales al museo, al incorporarlo a sus rutinas de ocio. Se perfila así como una herramienta útil de fidelización del público artístico. Además de que, al ser una opción ya elegida por ellos, donde ellos acuden o no voluntariamente, su parte de acción individual les vincula mucho más al proyecto, porque contamos con su participación activa desde su libertad.

Con las actividades propuestas desde el DEAC del MUSAC confluyen la escuela, el museo, la sociedad y el arte. Los institutos a través de sus profesores buscan la manera de acercar la 
realidad personal y social al aula con el eje vertebrador que son las obras de arte expuestas en el museo. Los alumnos reflexionan sobre la pandemia, el confinamiento y sus miedos y, a través de la expresión artística, lo hacen de una manera muy intuitiva y paralela. Esta crisis sanitaria a nivel mundial les ha dado una visión global de pertenencia, de sentido de humanidad, oportunidad para reflexionar y crecer espiritualmente desde los lenguajes de expresión y belleza de las obras de arte.

A nivel técnico, hemos comprobado que, pese al esfuerzo de la administración educativa de dotar a los centros de recursos tecnológicos, la realidad es que nos encontramos con una falta de estos que, en muchas ocasiones, es solventada por la buena voluntad de los docentes al hacer uso de sus propios recursos personales.

La tecnología es el canal, el medio del que disponemos para acortar distancias, pero no es la solución. La solución está en lo humano. Necesitamos algo que transforme el frío de las aulas con ventanas abiertas, alumnos sin rostro y continua desinfección antes y después de cada uso, en un espacio cálido y acogedor. Y ese algo que necesitamos es el arte, el museo de arte que entra a través de la pantalla para darnos calor, para transformar lo deshumanizado en humanizado y consolidar el arte como generador de un pensamiento crítico en nuestro alumnado, unido a las emociones.

Queremos resaltar, además, que los beneficios ocurren en todas las direcciones. No solo es un estímulo y un espacio de crecimiento para los estudiantes, sino que el museo, sin personas, no es, literalmente, nada. La propia existencia de los museos (desde la Revolución francesa) nace desde esa necesidad, abrirse a la sociedad. Los museos y sus profesionales necesitan ese mismo calor que demandamos desde las aulas.

En definitiva, necesitamos recuperar la visita presencial tan pronto como sea posible, pero sin olvidar el enorme potencial que supone para los centros educativos la utilización de la videollamada como recurso de primer orden para profundizar sobre los contenidos observados en el museo y que son trabajados de forma transversal por cada una de las materias que componen el currículo de la Educación Secundaria Obligatoria. De esta manera, incorporamos de forma sencilla, sin tener que desplazarnos del aula, componentes sumamente enriquecedores para el alumnado como son la novedad, la sorpresa, el pensamiento crítico, el autoconocimiento individual y colectivo, la autoexpresión, la materialización del pensamiento abstracto o la observación divergente, sensible y respetuosa de la realidad que habitamos. Gracias a este trabajo, reforzamos el vínculo entre el museo y los centros educativos, con un intercambio constante entre la investigación y la práctica, haciendo de la cooperación entre profesionales la piedra angular de nuestro día a día. 
Alicia Santos Álvarez, Julia R. Gallego García, M. Tránsito Domínguez Astorga y Gonzalo Marcos Treceño

\section{Referencias}

GARCÍA, A. (2017). Otra educación ya es posible: una introducción a las pedagogías alternativas. Barcelona: Litera Libros.

HERVÁS y Heras, J. (2014). El camino hacia la arquitectura:las mujeres de la Bauhaus. Tesis doctoral. Madrid: Universidad Politécnica de Madrid, <http://oa.upm.es/34242/> [Consulta: febrero 2021].

Huerta, R. y De La CAlle, R. (2008). Mentes sensibles. Investigar en educación y museos. Valencia: Universitat de València.

Jové, R. (2017). La escuela más feliz. Madrid: La esfera de los libros.

LABORATORIO DE INVESTIGACIÓN EDUCATIVA LAB. Qué-Por qué- Para quéCómo-Aprendizaje cooperativo. Propuesta para la implantación de una estructura de cooperación en el aula. $<$ https://labmadrid.com/wp-content/uploads/2016/03/Lab-01DOCUMENTACIO \%CC\%81N-APRENDIZAJE-COOPERATIVO.pdf $>$ [Consulta: febrero 2021]

LABRAdor Piquer, M.J. y ANDReu ANDrés, M.A. (eds.) (2008). Metodologías activas. Grupo de innovación en metodologías activas. Valencia: Universidad Politécnica de Valencia.

Mateu Mollá J. (2020). "Cómo afecta emocionalmente la crisis del coronavirus a los adolescentes" en The Conversation. $<$ https://theconversation.com/como-afectaemocionalmente-a-los-adolescentes-la-crisis-del-coronavirus-149770> [Consulta: enero 2021].

Pozuelos Estrada, F.J., Rodríguez Miranda, F. y García Prieto F.J. (2021). “Covid 19: 8 aprendizajes tras un año de escuela recluida" en The Conversation. España $<$ https://theconversation.com/covid-19-ocho-aprendizajes-tras-un-ano-de-escuela-recluida155166> [Consulta: 20 de febrero de 2021].

Priegue CaAmaño, D. y Crespo Comesaña, J.M. (2011). "El potencial pedagógico de la tecnología: desarrollar competencias y favorecer la autonomía y la responsabilidad en el alumnado". En XII Congreso Internacional de Teoría de la Educación. Barcelona: Universitat de Barcelona. Disponible en $<$ http://www.cite2011.com/Comunicaciones/TIC/112.pdf> [Consulta: febrero de 2021].

SAURA PÉREz, A. (2011). Innovación educativa con TIC en Educación artística, Plástica y visual. Madrid: MAD. 